\title{
MAGNETO-CONVECTIVE FLOWAND HEAT TRANSFER OF TWO IMMISCIBLE FLUIDS BETWEEN VERTICAL WAVY WALL AND A PARALLEL FLAT WALL
}

\author{
Mahadev M Biradar \\ Associate Professor, Dept. of Mathematics Basaveshwar Engineering College (Autonomous) Bagalkot, Karnataka INDIA \\ 587102
}

\begin{abstract}
Convective flow and heat transfer between vertical wavy wall and a parallel flat wall consisting of two regions, one filled with electrically conducting and other with viscous fluid is analyzed. Governing equation of motion have been solved by linearization technique. Results are presented for various parameters such as Hartmann number, Grashof number, viscosity ratio, width ratio, conductivity ratio and source or sink. The effect of all the parameters except the Hartmann number and source or sink remains same for two viscous immiscible fluids. The effect of Hartmann number is to decrease the velocity at the wavy and flat wall. The suppression near the flat wall compared to wavy wall is insignificant. The velocity is large for source compared to sink for equal and different wall temperature.
\end{abstract}

Keywords: Convection, vertical, wavy wall, immiscible

\section{INTRODUCTION}

Many transport process exists in natural and industrial applications in which the transfer of heat and mass occurs simultaneously as a result of buoyancy effect of thermal diffusion. Natural convection heat transfer plays an important role in the electronic components cooling since it has desirable characteristics in thermal equipments design; absence of mechanical or electromagnetic noise; low energy consumption, very important in portable computers; and reliability, since it has no elements to fail.

The optimization of the heat transfer has increasingly importance in electronic packaging due to the higher heat densities and to the electronic components and equipments miniaturization Sathe et.al. (1998). In spite of the abundant results about natural convection in electronic packaging, works dealing with heat transfer maximization is scarce in literature Landon et.al (1999) Da Silva et.al., (2004). This is, probably, due to the non-linear nature and to the difficult of natural convection simulation.

The corrugated wall channel is one of several devices employed for enhancing the heat transfer efficiency of industrial transport process. The problem of viscous flow in wavy channels was first treated analytically by Burns and Parks (1967) who expressed the stream function as a Fourier series under the assumption of stokes flow. Wang and Vanka (1995) determined the rates of heat transfer for flow through a periodic array of wavy passages. They observed that in the steady-flow regime, the average Nusselt numbers for the wavy-wall channel were only slightly larger than those for a parallel-plate channel. The problem of natural or mixed convection along a sinusoidal wavy surface extended previous work to complex geometries Yao (1983), Moulic et.al., $(1989,1989)$ and has received considerable attention due to its relevance to real geometries. An example of such geometry is a "roughened" surface that occurs often in problem involving the enhancement of heat transfer.

The flow and heat transfer of electrically conducting fluids in channels under the effect of a transverse magnetic field occurs in MHD-generators, pumps, accelerators, nuclear-reactors, filtration, geo-thermal system and others. Recently there are experimental and theoretical studies on hydromagnetic aspects of two fluid flows available in literature. Lohrasbi and Sahai (1998) dealt with two-phase magnetohydrodynamic (MHD) flow and heat transfer in a parallel plate channel. Two-phase MHD flow and heat transfer in an inclined channel is investigated by Malashetty and Umavathi (1997). Recently Malashetty et.al, $(2000,2001)$ analyzed the problem of fully developed two fluid magnetohydrodynamic flows with and without applied electric field in an inclined channel. Chamakha (2000) considered the steady, laminar flow of two viscous, incompressible electrically conducting and heat generating or absorbing immiscible fluids in an infinitely long, impermeable parallel-plate channel filled with a uniform porous medium. 
In spite of the numerous applications of corrugated walls, much work is not seen in literature. Hence it is the objective of the present work, is to study the problem of flow and heat transfer between vertical wavy wall and a parallel flat wall consisting of two regions, one filled with electrically conducting fluid and second with electrically non-conducting fluid.

\section{MATHEMATICAL FORMULATION}

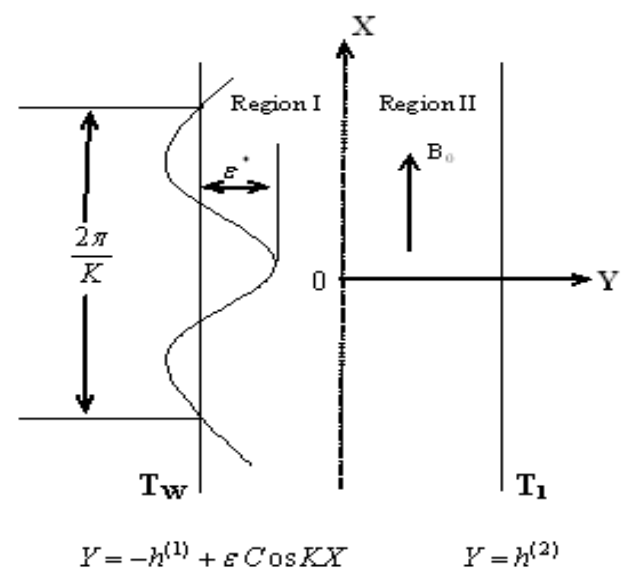

Fig-1: Physical Configuration

Consider the channel as shown in figure 4.1, in which the $X$ axis is taken vertically upwards and parallel to the flat wall while the $\mathrm{Y}$ axis is taken perpendicular to it in such a way that the wavy wall is represented by $Y=-h^{(1)}+\varepsilon * \cos K X$ and the flat wall by $Y=h^{(2)}$. The region $0 \leq y \leq h^{(1)}$ is occupied by viscous fluid of density $\rho^{(1)}$, viscosity $\mu^{(1)}$, thermal conductivity $k^{(1)}$ and the region $h^{(2)} \leq y \leq 0$ is occupied another viscous fluid of density $\rho^{(2)}$, viscosity $\mu^{(2)}$, thermal conductivity $k^{(2)}$. The wavy and flat walls are maintained at constant and different temperatures $T_{w}$ and $T_{1}$ respectively. We make the following assumptions:

(i) that all the fluid properties are constant except the density in the buoyancy-force term;

(ii) that the flow is laminar, steady and twodimensional;

(iii) that the viscous dissipation and the work done by pressure are sufficiently small in comparison with both the heat flow by conduction and the wall temperature;

(iv) that the wavelength of the wavy wall, which is proportional to $1 / \mathrm{K}$, is large.

Under these assumptions, the equations of momentum, continuity and energy which govern steady two-dimensional flow and heat transfer of viscous incompressible fluids are

\section{Region -I}

$\rho^{(1)}\left(U^{(1)} \frac{\partial U^{(1)}}{\partial X}+V^{(1)} \frac{\partial U^{(1)}}{\partial Y}\right)=-\frac{\partial P^{(1)}}{\partial X}+\mu^{(1)} \nabla^{2} U^{(1)}-\rho^{(1)} g$

$\rho^{(1)}\left(U^{(1)} \frac{\partial V^{(1)}}{\partial X}+V^{(1)} \frac{\partial V^{(1)}}{\partial Y}\right)=-\frac{\partial P^{(1)}}{\partial Y}+\mu^{(1)} \nabla^{2} V^{(1)}$

$\frac{\partial U^{(1)}}{\partial X}+\frac{\partial V^{(1)}}{\partial Y}=0$

$\rho^{(1)} C_{p}^{(1)}\left(U^{(1)} \frac{\partial T^{(1)}}{\partial X}+V^{(1)} \frac{\partial T^{(1)}}{\partial Y}\right)=k^{(1)} \nabla^{2} T^{(1)}+Q^{(1)}$

Region -II

$\rho^{(2)}\left(U^{(2)} \frac{\partial U^{(2)}}{\partial X}+V^{(2)} \frac{\partial U^{(2)}}{\partial Y}\right)=-\frac{\partial P^{(2)}}{\partial X}+\mu^{(2)} \nabla^{2} U^{(2)}$

$-\rho^{(2)} g-\sigma B_{0}^{2} U^{(2)}$

$\rho^{(2)}\left(U^{(2)} \frac{\partial V^{(2)}}{\partial X}+V^{(2)} \frac{\partial V^{(2)}}{\partial Y}\right)=-\frac{\partial P^{(2)}}{\partial Y}+\mu^{(2)} \nabla^{2} V^{(2)}$

$\frac{\partial U^{(2)}}{\partial X}+\frac{\partial V^{(2)}}{\partial Y}=0$

$\rho^{(2)} C_{p}^{(2)}\left(U^{(2)} \frac{\partial T^{(2)}}{\partial X}+V^{(2)} \frac{\partial T^{(2)}}{\partial Y}\right)=k^{(2)} \nabla^{2} T^{(2)}+Q^{(2)}$

Where the superscript indicates the quantities for regions I and II, respectively. To solve the above system of equations, one needs proper boundary and interface conditions. We assume $C_{p}^{(1)}=C_{p}^{(2)}$

The physical hydro dynamic conditions are

$$
\begin{aligned}
& U^{(1)}=0 \quad V^{(1)}=0, \quad \text { at } \quad Y=-h^{(1)}+\varepsilon^{*} \cos K X \\
& U^{(2)}=0 \quad V^{(2)}=0, \quad \text { at } \quad Y=h^{(2)} \\
& U^{(1)}=U^{(2)} V^{(1)}=V^{(2)}, \text { at } Y=0 \\
& \mu^{(1)}\left(\frac{\partial U}{\partial Y}+\frac{\partial V}{\partial X}\right)^{(1)}=\mu^{(2)}\left(\frac{\partial U}{\partial Y}+\frac{\partial V}{\partial X}\right)^{(2)} \text { at } Y=0
\end{aligned}
$$


The boundary and interface conditions on temperature are

$$
\begin{array}{llrlrl}
T^{(1)}=T_{W} & \text { at } & Y & =-h^{(1)}+\varepsilon^{*} \cos K X \\
T^{(2)}=T_{1} & \text { at } & Y & =h^{(2)} \\
T^{(1)}=T^{(2)} & \text { at } & Y & =0 \\
k^{(1)}\left(\frac{\partial T}{\partial Y}+\frac{\partial T}{\partial X}\right)^{(1)} & =k^{(2)}\left(\frac{\partial T}{\partial Y}+\frac{\partial T}{\partial X}\right)^{(2)} \text { at } Y=0
\end{array}
$$

The conditions on velocity represent the no-slip condition and continuity of velocity and shear stress across the interface. The conditions on temperature indicate that the plates are held at constant but different temperatures and continuity of heat and heat flux at the interface.

The basic equations (4.2.1) to (4.2.8) are made dimensionless using the following transformations

$$
\begin{aligned}
& (x, y)^{(1)}=\frac{1}{h^{(1)}}(X, Y)^{(1)} ;(u, v)^{(1)}=\frac{h^{(1)}}{v^{(1)}}(U, V)^{(1)} \\
& (x, y)^{(2)}=\frac{1}{h^{(2)}}(X, Y)^{(2)} ;(u, v)^{(2)}=\frac{h^{(2)}}{v^{(2)}}(U, V)^{(2)} \\
& \theta^{(1)}=\frac{\left(T^{(1)}-T_{s}\right)}{\left(T_{W}-T_{S}\right)} ; \quad \theta^{(2)}=\frac{\left(T^{(2)}-T_{s}\right)}{\left(T_{W}-T_{S}\right)} \\
& \bar{P}^{(1)}=\frac{P^{(1)}}{\rho\left(\frac{v^{(1)}}{h^{(1)}}\right)^{2}} ; \quad \bar{P}^{(2)}=\frac{P^{(2)}}{\rho\left(\frac{v^{(2)}}{h^{(2)}}\right)^{2}}
\end{aligned}
$$

Where $T_{s}$ is the fluid temperature in static conditions.

\section{Region -I}

$$
\begin{aligned}
& u^{(1)} \frac{\partial u^{(1)}}{\partial x}+v^{(1)} \frac{\partial u^{(1)}}{\partial y}=-\frac{\partial \bar{P}^{(1)}}{\partial x}+\frac{\partial^{2} u^{(1)}}{\partial x^{2}}+\frac{\partial^{2} u^{(1)}}{\partial y^{2}}+G \theta^{(1)} \\
& u^{(1)} \frac{\partial v^{(1)}}{\partial x}+v^{(1)} \frac{\partial v^{(1)}}{\partial y}=-\frac{\partial \bar{P}^{(1)}}{\partial y}+\frac{\partial^{2} v^{(1)}}{\partial x^{2}}+\frac{\partial^{2} v^{(1)}}{\partial y^{2}} \\
& \frac{\partial u^{(1)}}{\partial x}+\frac{\partial v^{(1)}}{\partial y}=0
\end{aligned}
$$

$$
u^{(1)} \frac{\partial \theta^{(1)}}{\partial x}+v^{(1)} \frac{\partial \theta^{(1)}}{\partial y}=\frac{1}{\operatorname{Pr}}\left(\frac{\partial^{2} \theta^{(1)}}{\partial x^{2}}+\frac{\partial^{2} \theta^{(1)}}{\partial y^{2}}\right)+\frac{\alpha}{\operatorname{Pr}}
$$

\section{Region -II}

$$
\begin{aligned}
& u^{(2)} \frac{\partial u^{(2)}}{\partial x}+v^{(2)} \frac{\partial u^{(2)}}{\partial y}=-\frac{\partial \bar{P}^{(2)}}{\partial x}+\frac{\partial^{2} u^{(2)}}{\partial x^{2}}+\frac{\partial^{2} u^{(2)}}{\partial y^{2}} \\
& +\beta m^{2} r^{2} h^{3} G \theta^{(2)}-M^{2} m h^{2} u^{(2)}
\end{aligned}
$$

$u^{(2)} \frac{\partial v^{(2)}}{\partial x}+v^{(2)} \frac{\partial v^{(2)}}{\partial y}=-\frac{\partial \bar{P}^{(2)}}{\partial y}+\frac{\partial^{2} v^{(2)}}{\partial x^{2}}+\frac{\partial^{2} v^{(2)}}{\partial y^{2}}$

$$
\begin{aligned}
& \frac{\partial u^{(2)}}{\partial x}+\frac{\partial v^{(2)}}{\partial y}=0 \\
& u^{(2)} \frac{\partial \theta^{(2)}}{\partial x}+v^{(2)} \frac{\partial \theta^{(2)}}{\partial y}=\frac{k m}{\operatorname{Pr}}\left(\frac{\partial^{2} \theta^{(2)}}{\partial x^{2}}+\frac{\partial^{2} \theta^{(2)}}{\partial y^{2}}\right) \\
& +\frac{m h^{2} \alpha Q}{\operatorname{Pr}}
\end{aligned}
$$

The dimensionless form of equation (2.9) and (2.10) using (2.11) become

$$
\begin{aligned}
& u^{(1)}=0 ; \quad v^{(1)}=0 \quad \text { at } y=-1+\varepsilon \cos \lambda x \\
& u^{(2)}=0 ; \quad v^{(2)}=0 \quad \text { at } y=1 \\
& u^{(1)}=\frac{1}{r m h} u^{(2)} ; v^{(1)}=\frac{1}{r m h} v^{(2)} \text { at } y=0 \\
& \left(\frac{\partial u}{\partial y}+\frac{\partial v}{\partial x}\right)^{(1)}=\frac{1}{r m^{2} h^{2}}\left(\frac{\partial u}{\partial y}+\frac{\partial v}{\partial x}\right)^{(2)} \text { at } y=0 \\
& \theta^{(1)}=1 \quad \text { at } \quad y=-1+\varepsilon \cos \lambda x \\
& \theta^{(2)}=\bar{\theta} \quad \text { at } y=1 \\
& \theta^{(1)}=\theta^{(2)} \quad \text { at } y=0
\end{aligned}
$$




$$
\left(\frac{\partial \theta}{\partial y}+\frac{\partial \theta}{\partial x}\right)^{(I)}=\frac{k}{h}\left(\frac{\partial \theta}{\partial y}+\frac{\partial \theta}{\partial x}\right)^{(2)} \text { at } y=0
$$

\section{SOLUTIONS}

The governing equations (2.12) to (2.15), (2.16) to (2.19) along with boundary and interface conditions (2.20) to (2.21) are two dimensional, nonlinear and coupled and hence closed form solutions can not be found. However approximate solutions can be found using the method of regular perturbation. We take flow field and the temperature field to be

\section{Region-I}

$$
\begin{aligned}
& u^{(1)}(x, y)=u_{0}^{(1)}(y)+\varepsilon u_{l}^{(1)}(x, y) \\
& v^{(1)}(x, y)=\varepsilon v_{l}^{(1)}(x, y) \\
& \bar{P}^{(1)}(x, y)=P_{0}^{(1)}(x)+\varepsilon P_{1}^{(1)}(x, y) \\
& \theta^{(1)}(x, y)=\theta_{0}^{(1)}(y)+\varepsilon \theta_{1}^{(1)}(x, y)
\end{aligned}
$$

\section{Region -II}

$$
\begin{aligned}
& u^{(2)}(x, y)=u_{0}^{(2)}(y)+\varepsilon u_{l}^{(2)}(x, y) \\
& v^{(2)}(x, y)=\varepsilon v_{l}^{(2)}(x, y) \\
& \bar{P}^{(2)}(x, y)=P_{0}^{(2)}(x)+\varepsilon P_{1}^{(2)}(x, y) \\
& \theta^{(2)}(x, y)=\theta_{0}^{(2)}(y)+\varepsilon \theta_{l}^{(2)}(x, y)
\end{aligned}
$$

Where the perturbations $u_{1}^{(i)}, v_{1}^{(i)}, P_{1}^{(i)}$ and $\theta_{1}^{(i)}$ for $i=1,2$ are small compared with the mean or the zeroth order quantities. Using equation (4.3.1) and (4.3.2) in the equation (2.12) to (2.15), (2.16) to (2.19) become

\subsection{Zeroth Order}

\section{Region-I}

$\frac{d^{2} u_{0}^{(1)}}{d y^{2}}=-G \theta_{0}^{(1)}$

$\frac{d^{2} \theta_{0}^{(1)}}{d y^{2}}=-\alpha$

\section{Region-II}

$\frac{d^{2} u_{0}^{(2)}}{d y^{2}}-m h^{2} M^{2} u_{0}^{(2)}=-\beta m^{2} r^{2} h^{3} G \theta_{0}^{(2)}$

$\frac{d^{2} \theta_{0}^{(2)}}{d y^{2}}=\frac{-\alpha Q h^{2}}{k}$

\subsection{First Order:}

\section{Region-I}

$$
\begin{aligned}
& u_{0}^{(1)} \frac{\partial u_{1}^{(1)}}{\partial x}+v_{1}^{(1)} \frac{d u_{0}^{(1)}}{d y}=-\frac{\partial P_{1}^{(1)}}{\partial x}+\frac{\partial^{2} u_{1}^{(1)}}{\partial x^{2}}+\frac{\partial^{2} u_{1}^{(1)}}{\partial y^{2}}+G \theta_{1}^{(1)} \\
& u_{0}^{(1)} \frac{\partial v_{1}^{(1)}}{\partial x}=-\frac{\partial P_{1}^{(1)}}{\partial y}+\frac{\partial^{2} v_{1}^{(1)}}{\partial x^{2}}+\frac{\partial^{2} v_{1}^{(1)}}{\partial y^{2}} \\
& \frac{\partial u_{1}^{(1)}}{\partial x}+\frac{\partial^{2} v_{1}^{(1)}}{\partial y^{2}}=0
\end{aligned}
$$

$$
\operatorname{Pr}\left(u_{0}^{(1)} \frac{\partial \theta_{1}^{(1)}}{\partial x}+v_{1}^{(1)} \frac{d \theta_{0}^{(1)}}{d y}\right)=\frac{\partial^{2} \theta_{1}^{(1)}}{\partial x^{2}}+\frac{\partial^{2} \theta_{1}^{(1)}}{\partial y^{2}}
$$

\section{Region-II}

$$
\begin{aligned}
& u_{0}^{(2)} \frac{\partial u_{1}^{(2)}}{\partial x}+v_{1}^{(2)} \frac{d u_{0}^{(2)}}{d y}=-\frac{\partial P_{1}^{(2)}}{\partial x}+\frac{\partial^{2} u_{1}^{(2)}}{\partial x^{2}}+\frac{\partial^{2} u_{1}^{(2)}}{\partial y^{2}} \\
& +\beta m^{2} r^{2} h^{3} G \theta_{1}^{(2)}-m h^{2} M^{2} u_{1}^{(2)}
\end{aligned}
$$

$u_{0}^{(2)} \frac{\partial v_{1}^{(2)}}{\partial x}=-\frac{\partial P_{1}^{(2)}}{\partial y}+\frac{\partial^{2} v_{1}^{(2)}}{\partial x^{2}}+\frac{\partial^{2} v_{1}^{(2)}}{\partial y^{2}}$

$\frac{\partial u_{l}^{(2)}}{\partial x}+\frac{\partial^{2} v_{l}^{(2)}}{\partial y^{2}}=0$

$u_{0}^{(2)} \frac{\partial \theta_{1}^{(2)}}{\partial x}+v_{1}^{(2)} \frac{d \theta_{0}^{(2)}}{d y}=\frac{k m}{\operatorname{Pr}}\left(\frac{\partial^{2} \theta_{1}^{(2)}}{\partial x^{2}}+\frac{\partial^{2} \theta_{1}^{(2)}}{\partial y^{2}}\right)$ 
Where $\quad C^{(1)}=\frac{\partial\left(P_{0}-P_{S}\right)^{(1)}}{\partial x}$ and $\quad C^{(2)}=\frac{\partial\left(P_{0}-P_{S}\right)^{(2)}}{\partial x}$ taken equal to zero (see Ostrach, 1952). With the help of (3.1) and (3.2) the boundary and interface conditions (2.20) to (2.21) become

$$
\begin{array}{ll}
u_{0}^{(1)}(-1)=0 & \text { at } y=-1 \\
u_{0}^{(2)}(1)=0 & \text { at } y=1 \\
u_{0}^{(1)}=\frac{1}{r m h} u_{0}^{(2)} & \text { at } y=0 \\
\frac{d u_{0}^{(1)}}{d y}=\frac{1}{r m^{2} h^{2}} \frac{d u_{0}^{(2)}}{d y} & \text { at } y=0 \\
\theta_{0}^{(1)}(-1)=1 & \text { at } y=-1 \\
\theta_{0}^{(2)}(1)=\bar{\theta} & \text { at } y=0 \\
\theta_{0}^{(1)}=\theta_{0}^{(2)} & \text { at } y=0 \\
\frac{d \theta_{0}^{(1)}}{d y}=\frac{k}{h} \frac{d \theta_{0}^{(2)}}{d y} &
\end{array}
$$

$u_{1}^{(1)}(-1)=-\operatorname{Cos} \lambda x \frac{d u_{0}^{(1)}}{d y} ; v_{1}^{(1)}(-1)=0$ at $y=-1$

$u_{1}^{(2)}(1)=0 ; v_{1}^{(2)}(1)=0 \quad$ at $y=1$

$\frac{\partial u_{1}^{(1)}}{\partial y}+\frac{\partial v_{1}^{(1)}}{\partial x}=\frac{1}{r m^{2} h^{2}}\left(\frac{\partial u_{1}^{(2)}}{\partial y}+\frac{\partial v_{1}^{(2)}}{\partial x}\right)$ at $y=0$

$$
\theta_{1}^{(1)}(-1)=-\operatorname{Cos} \lambda x \frac{d \theta_{0}^{(1)}}{d y} \quad \text { at } y=-1
$$

$$
\theta_{1}^{(2)}(1)=0 \quad \text { at } y=1
$$

$$
\begin{aligned}
& \theta_{1}^{(1)}=\theta_{1}^{(2)} \quad \text { at } y=0 \\
& \frac{\partial \theta_{1}^{(1)}}{\partial y}+\frac{\partial \theta_{1}^{(1)}}{\partial x}=\frac{k}{h}\left(\frac{\partial \theta_{1}^{(2)}}{\partial y}+\frac{\partial \theta_{1}^{(2)}}{\partial x}\right) \text { at } y=0
\end{aligned}
$$

Introducing the stream function $\bar{\psi}_{1}$ defined by

$$
u_{1}^{(1)}=-\frac{\partial \bar{\psi}_{1}^{(1)}}{\partial y} ; v_{1}^{(1)}=\frac{\partial \bar{\psi}_{1}^{(1)}}{\partial x}
$$

And eliminating $P_{1}^{(1)}$ and $P_{1}^{(2)}$ from equation (3.7), (3.8) and (3.11), (3.12) we get

\section{Region-I}

$$
\begin{aligned}
& u_{0}^{(1)}\left(\frac{\partial^{3} \bar{\psi}_{1}^{(1)}}{\partial x \partial y^{2}}+\frac{\partial^{3} \bar{\psi}_{1}^{(1)}}{\partial x^{3}}\right)-\frac{\partial \bar{\psi}_{1}^{(1)}}{\partial x} \frac{d^{2} u_{0}^{(1)}}{d y^{2}}= \\
& 2 \frac{\partial^{4} \bar{\psi}_{1}^{(1)}}{\partial x^{2} \partial y^{2}}+\frac{\partial^{4} \bar{\psi}_{1}^{(1)}}{\partial x^{4}}+\frac{\partial^{4} \bar{\psi}_{1}^{(1)}}{\partial y^{4}}-G \frac{\partial \theta_{1}^{(1)}}{\partial y}
\end{aligned}
$$

$\operatorname{Pr}\left(u_{0}^{(1)} \frac{\partial \theta_{1}^{(1)}}{\partial x}+\frac{\partial \bar{\psi}_{1}^{(1)}}{\partial x} \frac{d \theta_{0}^{(1)}}{d y}\right)=\frac{\partial^{2} \theta_{1}^{(1)}}{\partial x^{2}}+\frac{\partial^{2} \theta_{1}^{(1)}}{\partial y^{2}}$

\section{Region-II}

$$
\begin{aligned}
& u_{0}^{(2)}\left(\frac{\partial^{3} \bar{\psi}_{1}^{(2)}}{\partial x \partial y^{2}}+\frac{\partial^{3} \bar{\psi}_{1}^{(2)}}{\partial x^{3}}\right)-\frac{\partial \bar{\psi}_{1}^{(2)}}{\partial x} \frac{d^{2} u_{0}^{(2)}}{d y^{2}}=2 \frac{\partial^{4} \bar{\psi}_{1}^{(2)}}{\partial x^{2} \partial y^{2}} \\
& +\frac{\partial^{4} \bar{\psi}_{1}^{(2)}}{\partial x^{4}}+\frac{\partial^{4} \bar{\psi}_{1}^{(2)}}{\partial y^{4}}-\beta m^{2} r^{2} h^{3} G \frac{\partial \theta_{1}^{(2)}}{\partial y}-m h^{2} M^{2} \frac{\partial^{2} \psi_{1}}{\partial y^{2}}
\end{aligned}
$$

$$
u_{0}^{(2)} \frac{\partial \theta_{1}^{(2)}}{\partial x}+\frac{\partial \bar{\psi}_{1}^{(2)}}{\partial x} \frac{d \theta_{0}^{(2)}}{d y}=\frac{k m}{\operatorname{Pr}}\left(\frac{\partial^{2} \theta_{1}^{(1)}}{\partial x^{2}}+\frac{\partial^{2} \theta_{1}^{(1)}}{\partial y^{2}}\right)
$$

Assuming

$$
\bar{\psi}_{1}^{(i)}(x, y)=\varepsilon e^{i \lambda x} \psi^{(i)}(y), \theta_{1}^{(i)}(x, y)=\varepsilon e^{i \lambda x} t^{(i)}(y)
$$$$
\text { for } \mathrm{i}=1,2
$$

From which we infer

$$
\begin{aligned}
& u_{1}^{(i)}(x, y)=\varepsilon e^{i \lambda x} \bar{u}_{1}^{(i)}(y), v_{1}^{(i)}(x, y)=\varepsilon e^{i \lambda x} \bar{v}_{1}^{(i)}(y) \\
& \text { for } \mathrm{i}=1,2
\end{aligned}
$$


And using (3.24) in (3.20) to (3.23), we get

\section{Region-I}

$$
\begin{aligned}
& \psi^{i v}-i \lambda\left[u_{0}^{(1)}\left(\psi^{\prime \prime}-\lambda^{2} \psi\right)-\frac{d^{2} u_{0}^{(1)}}{d y^{2}} \psi\right]^{(1)} \\
& -\lambda^{2}\left(2 \psi^{\prime \prime}-\lambda^{2} \psi\right)=G \frac{d t^{(1)}}{d y}
\end{aligned}
$$

$t^{\prime \prime}-\lambda^{2} t=\operatorname{Pr} i \lambda\left(u_{0} t+\psi \frac{d \theta_{0}}{d y}\right)^{(1)}$

$$
\begin{array}{ll}
t^{(1)}=-\frac{d \theta_{0}^{(1)}}{d y} & \text { at } y=-1 \\
t^{(2)}=0 & \text { at } y=1 \\
t^{(1)}=t^{(2)} & \text { at } y=0 \text { for } i \geq 1 \\
\frac{d t^{(1)}}{d y}=\frac{d t^{(2)}}{d y} & \text { at } y=0 \quad
\end{array}
$$

\section{Region-II}

$$
\begin{aligned}
& \psi^{i v}-i \lambda\left[u_{0}^{(2)}\left(\psi^{\prime \prime}-\lambda^{2} \psi\right)-\frac{d^{2} u_{0}^{(2)}}{d y^{2}} \psi\right]^{(2)}-m h^{2} M^{2} \psi^{\prime \prime} \\
& -\lambda^{2}\left(2 \psi^{\prime \prime}-\lambda^{2} \psi\right)=\beta m^{2} r^{2} h^{3} G \frac{d t^{(2)}}{d y}
\end{aligned}
$$

$$
t^{\prime \prime}-\lambda^{2} t=\frac{\operatorname{Pr}}{k m} i \lambda\left(u_{0} t+\psi \frac{d \theta_{0}}{d y}\right)^{(2)}
$$

Below we restrict our attention to the real parts of the solution for the perturbed quantities $\bar{\psi}_{1}^{(i)}, \theta_{1}^{(i)}, u_{1}^{(i)}$ and $v_{1}^{(i)}$ for $i=1,2$

The boundary conditions (3.17) and (3.18) can be now written in terms of

$$
\frac{\partial \bar{\psi}_{1}^{(1)}}{\partial y}=-\operatorname{Cos} \lambda x \frac{d u_{0}^{(1)}}{d y} ; \quad \frac{\partial \bar{\psi}_{1}^{(1)}}{\partial x}=0 \quad \text { at } \quad y=-1
$$

$$
\frac{d^{4} \psi_{1}^{(1)}}{d y^{4}}-i\left(u_{0} \frac{d^{2} \psi_{0}}{d y^{2}}-\frac{d^{2} u_{0}}{d y^{2}} \psi_{0}\right)^{(1)}=G \frac{d t_{1}^{(1)}}{d y}
$$

$$
\frac{d^{2} t_{0}^{(1)}}{d y^{2}}=0
$$

$$
\frac{\partial \bar{\psi}_{1}^{(2)}}{\partial y}=0 ; \quad \frac{\partial \bar{\psi}_{1}^{(2)}}{\partial x}=0 \quad \text { at } \quad y=1
$$

$$
\frac{\partial \bar{\psi}_{1}^{(1)}}{\partial y}=\frac{1}{r m h} \frac{\partial \bar{\psi}_{1}^{(2)}}{\partial y} ; \frac{\partial \bar{\psi}_{1}^{(1)}}{\partial x}=\frac{1}{r m h} \frac{\partial \bar{\psi}_{1}^{(2)}}{\partial x}
$$

$$
\frac{d^{2} t_{1}^{(1)}}{d y^{2}}=\operatorname{Pr} i\left(u_{0} t_{0}+\psi_{0} \frac{d \theta_{0}}{d y}\right)^{(1)}
$$

\section{Region-II}

$$
\frac{d^{4} \psi_{0}^{(2)}}{d y^{4}}-M^{2} m h^{2} \frac{d^{2} \psi_{0}^{(2)}}{d y^{2}}=\beta m^{2} r^{2} h^{3} G \frac{d t_{0}^{(2)}}{d y}
$$

$$
\text { at } y=0
$$

$$
\frac{\partial^{2} \bar{\psi}_{1}^{(1)}}{\partial y^{2}}+\frac{\partial^{2} \bar{\psi}_{1}^{(1)}}{\partial x^{2}}=\frac{1}{r m^{2} h^{2}}\left(\frac{\partial^{2} \bar{\psi}_{1}^{(2)}}{\partial y^{2}}+\frac{\partial^{2} \bar{\psi}_{1}^{(2)}}{\partial x^{2}}\right)
$$$$
\frac{d^{2} t_{0}^{(2)}}{d y^{2}}=0
$$ 


$$
\begin{aligned}
& \frac{d^{4} \psi_{1}^{(2)}}{d y^{4}}-M^{2} m h^{2} \frac{d^{2} \psi_{1}^{(2)}}{d y^{2}}=i\left(u_{0} \frac{d^{2} \psi_{0}}{d y^{2}}-\frac{d^{2} u_{0}}{d y^{2}} \psi_{0}\right)^{(2)} \\
& +\beta m^{2} r^{2} h^{3} G \frac{d t_{1}^{(2)}}{d y} \\
& \frac{d^{2} t_{1}^{(2)}}{d y^{2}}=\frac{\operatorname{Pr}}{k m} i\left(u_{0} t_{0}+\psi_{0} \frac{d \theta_{0}}{d y}\right)^{(2)}
\end{aligned}
$$

and

$$
\begin{array}{ll}
\frac{d \psi_{0}^{(1)}}{d y}=\operatorname{Cos} \lambda x \frac{d u_{0}^{(1)}}{d y} ; \quad \psi_{0}^{(1)}=0 & \text { at } \quad y=-1 \\
\frac{d \psi_{0}^{(2)}}{d y}=0 ; \quad \psi_{0}^{(2)}=0 & \text { at } \quad y=1
\end{array}
$$$$
\frac{d \psi_{0}^{(1)}}{d y}=\frac{1}{r m h} \frac{d \psi_{0}^{(2)}}{d y} ; \quad \psi_{0}^{(1)}=\frac{1}{r m h} \psi_{0}^{(2)} \text { at } y=0
$$$$
\frac{d^{2} \psi_{0}^{(1)}}{d y^{2}}=\frac{1}{r m^{2} h^{2}} \frac{d^{2} \psi_{0}^{(2)}}{d y^{2}} ; \psi_{0}^{(1)}=\frac{1}{r m^{2} h^{2}} \psi_{0}^{(2)}
$$

at $y=0$

$$
\frac{d \psi_{1}^{(1)}}{d y}=0 ; \quad \psi_{1}^{(1)}=0 \quad \text { at } \quad y=-1
$$

$$
\frac{d \psi_{1}^{(2)}}{d y}=0 ; \quad \psi_{1}^{(2)}=0 \quad \text { at } \quad y=1
$$$$
\frac{d \psi_{1}^{(1)}}{d y}=\frac{1}{r m h} \frac{d \psi_{1}^{(2)}}{d y} ; \quad \psi_{1}^{(1)}=\frac{1}{r m h} \psi_{1}^{(2)} \text { at } y=0
$$$$
\frac{d^{2} \psi_{1}^{(1)}}{d y^{2}}=\frac{1}{r m^{2} h^{2}} \frac{d^{2} \psi_{1}^{(2)}}{d y^{2}} ; \psi_{1}^{(1)}=\frac{1}{r m^{2} h^{2}} \psi_{1}^{(2)}
$$

at $y=0$

$t_{0}^{(1)}=-\frac{d \theta_{0}^{(1)}}{d y}$

at $y=-1$

$t_{0}^{(2)}=0$

at $y=1$

$t_{0}^{(1)}=t_{0}^{(2)}$

at $y=0$

$$
\frac{d t_{0}^{(1)}}{d y}=\frac{k}{h} \frac{d t_{0}^{(2)}}{d y} \quad \text { at } y=0
$$

$$
\begin{array}{ll}
t_{1}^{(1)}=0 & \text { at } y=-1 \\
t_{1}^{(2)}=0 & \text { at } y=1
\end{array}
$$

$$
t_{1}^{(1)}=t_{1}^{(2)}
$$

at $y=0$ for $i \geq 1$

$$
\frac{d t_{1}^{(1)}}{d y}=\frac{d t_{1}^{(2)}}{d y} \quad \text { at } y=0
$$

\subsection{Zeroth-Order Solution (Mean Part)}

The solutions to zeroth order differential Eqs. (3.3) to (3.6) using boundary and interface conditions (3.15) and (3.16) are given by

$$
t_{0}^{(1)}=C_{5} y+C_{6}
$$

\section{Region-I}

$$
\begin{aligned}
& u_{0}^{(1)}=l_{1} y^{4}+l_{2} y^{3}+l_{3} y^{2}+A_{1} y+A_{2} \\
& \theta_{0}^{(1)}=d_{1} y^{2}+C_{1} y+C_{2}
\end{aligned}
$$

\section{Region-II}

$u_{0}^{(2)}=B_{1} \cosh n y+B_{2} \sinh n y+s_{1} y^{2}+s_{2} y+s_{3}$

$$
\theta_{0}^{(2)}=f_{2} y^{2}+C_{3} y+C_{4}
$$

The solutions of zeroth and first order of $\lambda$ are obtained by solving the equation (3.33) to (3.40) using boundary and interface conditions (3.41) and (3.42) and are given below

$\psi_{0}^{(1)}=l_{4} y^{4}+\frac{A_{3}}{6} y^{3}+\frac{A_{4}}{2} y^{2}+A_{5} y+A_{6}$

$\psi_{0}^{(2)}=B_{3} \cosh n y+B_{4} \sinh n y+B_{5} y+B_{6}+s_{4} y^{2}$

$t_{0}^{(2)}=C_{7} y+C_{8}$

$\psi_{1}^{(1)}=i\left(l_{20} y^{10}+l_{21} y^{9}+l_{22} y^{8}+l_{23} y^{7}+l_{24} y^{6}+l_{25} y^{5}+l_{26} y^{4}\right)$

$+\frac{A_{7}}{6} y^{3}+\frac{A_{8}}{2} y^{2}+A_{9} y+A_{10}$ 


$$
\begin{aligned}
& t_{1}^{(1)}=i \operatorname{Pr}\left(d_{8} y^{7}+d_{9} y^{6}+d_{10} y^{5}+d_{11} y^{4}+d_{12} y^{3}+d_{13} y^{2}\right) \\
& +q_{6} y+q_{8} \\
& \psi_{1}^{(2)}=B_{7} \cosh n y+B_{8} \sinh n y+B_{7} y+B_{10}+ \\
& i\left(s_{29} y^{3} \cosh n y+s_{30} y^{3} \sinh n y+s_{31} y^{2} \cosh n y\right. \\
& +s_{32} y^{2} \sinh n y+s_{33} y \cosh n y+s_{34} y \sinh n y+ \\
& s_{35} \cosh n y+s_{36} \sinh n y+S_{37} y^{6}+S_{38} y^{5}+ \\
& \left.S_{39} y^{4}+S_{40} y^{3}+S_{41} y^{2}\right) \\
& t_{1}^{(2)}=i \frac{\operatorname{Pr}}{k m}\left(f_{11} y \cosh n y+f_{12} y \sinh n y+f_{13} \cosh n y\right. \\
& \left.+f_{14} \sinh n y+f_{15} y^{5}+f_{16} y^{4}+f_{17} y^{3}+f_{18} y^{2}\right) \\
& +q_{5} y+q_{7}
\end{aligned}
$$

The first order quantities can be put in the forms

$$
u_{1}=\psi_{i}^{\prime} \sin \lambda x-\psi_{r}^{\prime} \cos \lambda x
$$$$
v_{1}=\lambda \psi_{r}^{\prime} \sin \lambda x-\lambda \psi_{i}^{\prime} \cos \lambda x
$$

$\theta_{1}=t_{r} \cos \lambda x-t_{i} \sin \lambda x$

\section{Region-I}

$$
\begin{aligned}
& u_{1}^{(1)}=-\cos \lambda x\left(\psi_{0 r}^{\prime}+\lambda \psi_{1 r}^{\prime}\right)^{(1)}+\sin \lambda x\left(\psi_{0 i}^{\prime}+\lambda \psi_{1 i}^{\prime}\right)^{(1)} \\
& v_{1}^{(1)}=-\lambda \cos \lambda x\left(\psi_{0 i}+\lambda \psi_{1 i}\right)^{(1)}-\lambda \sin \lambda x\left(\psi_{0 r}+\lambda \psi_{1 r}\right)^{(1)} \\
& \theta_{1}^{(1)}=\cos \lambda x\left(t_{0 r}+\lambda t_{1 r}\right)^{(1)}-\sin \lambda x\left(t_{0 i}+\lambda t_{1 i}\right)^{(1)}
\end{aligned}
$$

\section{Region-II}

$$
\begin{aligned}
& u_{1}^{(2)}=-\cos \lambda x\left(\psi_{0 r}^{\prime}+\lambda \psi_{1 r}^{\prime}\right)^{(2)}+\sin \lambda x\left(\psi_{0 i}^{\prime}+\lambda \psi_{1 i}^{\prime}\right)^{(2)} \\
& v_{1}^{(2)}=-\lambda \cos \lambda x\left(\psi_{0 i}+\lambda \psi_{1 i}\right)^{(2)}-\lambda \sin \lambda x\left(\psi_{0 r}+\lambda \psi_{1 r}\right)^{(2)} \\
& \theta_{1}^{(2)}=\cos \lambda x\left(t_{0 r}+\lambda t_{1 r}\right)^{(2)}-\sin \lambda x\left(t_{0 i}+\lambda t_{1 i}\right)^{(2)}
\end{aligned}
$$

\section{RESULTS AND DISCUSSION}

\subsection{Discussion of the Zeroth Order Solution:}

The effect of Hartmann number M on zeroth order velocity is to decrease the velocity for $\bar{\theta}=0, \pm 1$, but the suppression is more effective near the flat wall as $\mathrm{M}$ increases as shown in figure 2 .

The effect of heat source $(\alpha>0)$ or $\operatorname{sink}(\alpha<0)$ and in the absence of heat source or sink $(\alpha=0)$, on zeroth order velocity is shown in figure 3 for $\bar{\theta}=0, \pm 1$. It is observed that heat source promote the flow, sink suppress the flow, and the velocity profiles lie in between source or sink for $\alpha=0$. We also observe that the magnitude of zeroth order velocity is optimum for $\bar{\theta}=1$ and minimal for $\bar{\theta}=-1$, and profiles lies between $\bar{\theta}= \pm 1$ for $\bar{\theta}=0$. The effect of source or sink parameter $\alpha$ on zeroth order temperature is similar to that on zeroth order velocity as shown in figure 4 . The effect of free convection parameter $\mathrm{G}$, viscosity ratio $\mathrm{m}$, width ratio $\mathrm{h}$, on zeroth order velocity and the effect of width ratio $h$, conductivity ratio $\mathrm{k}$, on zeroth order temperature remain the same as explained in chapter-III

The effect of free convection parameter $G$ on first order velocity is shown in figure.5. As $G$ increases $u_{1}$ increases near the wavy and flat wall where as it deceases at the interface and the suppression is effective near the flat wall for $\bar{\theta}=0, \pm 1$. The effect of viscosity ratio $\mathrm{m}$ on $\mathrm{u}_{1}$ shows that as $\mathrm{m}$ increases first order velocity increases near the wavy and flat wall, but the magnitude is very large near flat wall . At the interface velocity decreases as $\mathrm{m}$ increases and the suppression is significant towards the flat wall as seen in figure 6 for $\bar{\theta}=0, \pm 1$. The effect of width ratio $\mathrm{h}$ on first order velocity $\mathrm{u}_{1}$ shows that $\mathrm{u}_{1}$ remains almost same for $\mathrm{h}<1$ but is more effective for $\mathrm{h}>1$. For $\mathrm{h}=2, \mathrm{u}_{1}$ increases near the wavy and flat wall and drops at the interface, for $\bar{\theta}=0, \pm 1$ as seen in figure 7.

The effect of Hartmann number $\mathrm{M}$ on $\mathrm{u}_{1}$ shows that as $\mathrm{M}$ increases velocity decreases at the wavy wall and the flat wall but the suppression near the flat wall compared to wavy wall is insignificant and as $\mathrm{M}$ increases $\mathrm{u}_{1}$ increases in magnitude at the interface for $\bar{\theta}=0, \pm 1$ as seen in figure 8 . The effect of $\alpha$ on $\mathrm{u}_{1}$ is shown in figure 9 , which shows that velocity is large near the wavy and flat wall for heat source $\alpha=5$ and is less for heat $\operatorname{sink} \alpha=-5$. Similar result is obtained at the interface but for negative values of $\mathrm{u}_{1}$. Here also we observe that the magnitude is very large near the wavy wall compared to flat wall. 
The effect of convection parameter $\mathrm{G}$, viscosity ratio $\mathrm{m}$, width ratio $h$, thermal conductivity ratio $k$ on first order velocity $v_{1}$ is similar as explained in chapter III. The effect of Hartmann number $\mathrm{M}$ is to increase the velocity near the wavy wall and decrease velocity near the flat wall for $\bar{\theta}=0, \pm 1$, whose results are applicable to flow reversal problems as shown in figure.10. The effect of source or sink on first order velocity $v_{1}$ is shown in figure 11 . For heat source, $v_{1}$ is less near wavy wall and more for flat wall where as we obtained the opposite result for sink i.e. $\mathrm{v}_{1}$ is maximum near wavy wall and minimum near the flat wall, for $\alpha=0$ the profiles lie in between $\alpha= \pm 5$

The effect of convection parameter $\mathrm{G}$, viscosity ratio $\mathrm{m}$, width ratio $\mathrm{h}$, thermal conductivity ratio $\mathrm{k}$, Hartmann number and source and sink parameter $\alpha$ on first order temperature are shown in figures 12 to 17 . It is seen that $\mathrm{G}, \mathrm{m}, \mathrm{h}$, and $\mathrm{k}$ increases in magnitude for values of $\bar{\theta}=0, \pm 1$. It is seen that from figure 16 that as $\mathrm{M}$ increases the magnitude of $\theta_{1}$ decreases for $\bar{\theta}=0, \pm 1$. Figure 17 shows that the magnitude of $\alpha$ is large for heat source and is less for sink whereas $\theta_{1}$ remains invariant for $\alpha=0$.

The effect of convection parameter $\mathrm{G}$, viscosity ratio $\mathrm{m}$, width ratio $\mathrm{h}$, and thermal conductivity ratio $\mathrm{k}$, on total velocity remains the same as explained in chapter III. The effect of heat source or sink on total velocity shows that $\mathrm{U}$ is very large for $\alpha=5$ compared to $\alpha=-5$ and is almost invariant for $\alpha=0$ as seen figure 18 , for all values of $\bar{\theta}$.

The effect of Grashof number $\mathrm{G}$, viscosity ratio $\mathrm{m}$, shows that increasing $\mathrm{G}$ and $\mathrm{m}$ suppress the total temperature but the supression for $\mathrm{m}$ is negligible as seen in figures.19 and 20 . The effects of width ratio $h$ and conductivity ratio $k$ is same as explained in chapter-III. The effect of source or sink parameter on total temperature remains the same as that on total velocity as seen figure. 21 .

Where

$$
\begin{aligned}
& d_{1}=-\alpha / 2 ; \quad f_{1}=-\alpha Q h^{2} / k ; \quad f_{2}=f_{1} / 2 \\
& C_{3}=-\frac{\left(1-\theta-d_{1}+f_{2}\right) h}{(k+h)} ; \quad C_{4}=\theta-C_{3}-f_{2} ; \\
& C_{2}=C_{4} C_{1}=C_{2}-1+d_{1} ; \quad l_{1}=-\frac{G d_{1}}{12} ; \\
& l_{2}=-\frac{G C_{1}}{6} ; \quad l_{3}=-\frac{G C_{2}}{2} s_{1}=\frac{\beta m^{2} r^{2} h^{3} G f_{2}}{n^{2}} ;
\end{aligned}
$$

$$
\begin{aligned}
& s_{2}=\frac{\beta m^{2} r^{2} h^{3} G C_{3}}{n^{2}} s_{3}=\frac{2 \beta m^{2} r^{2} h^{3} G f_{2}}{n^{4}}+\frac{\beta m^{2} r^{2} h^{3} G C_{4}}{n^{2}} \\
& l_{4}=\frac{G C_{5}}{24} ; \quad s_{4}=-\frac{\beta m^{2} r^{2} h^{3} \cdot G_{7}}{n^{2}} \quad l_{5}=\frac{6+n^{2}}{r m^{2} h^{2}} ; \quad l_{6}=-\frac{4 n}{r m h} ; \\
& l_{7}=-\frac{4}{r m h} ; \quad l_{8}=\frac{6}{r m^{2} h^{2}} \\
& l_{9}=2 A_{1}+2 l_{4}-\frac{6 s_{4}}{r m^{2} h^{2}}-8 l_{1}+6 l_{2}-4 l_{3} \\
& l_{10}=(\cosh n-n \sinh n)\left(l_{7} n \cosh n-l_{6}\right)- \\
& \left(l_{7} n \sinh n-l_{5}\right)(\sinh n-n \cosh n) \\
& l_{11}=\left(l_{7} n \cosh n-l_{6}\right)+l_{8}(\sinh n-n \cosh n) \\
& l_{12}=l_{9}(\sinh n-n \cosh n)-s_{4}\left(l_{7} n \cosh n-l_{6}\right) \\
& d_{2}=l_{1} C_{5}-\alpha l_{4} ; \quad d_{3}=l_{2} C_{5}+l_{1} C_{6}-\frac{\alpha A_{3}}{6}+C_{1} l_{4} \\
& d_{4}=l_{3} C_{5}+l_{2} C_{8}-\frac{\alpha A_{4}}{2}+\frac{C_{1} A_{3}}{6} \\
& d_{5}=A_{1} C_{5}+l_{3} C_{6}-\alpha A_{4}+\frac{C_{1} A_{4}}{2} \\
& d_{6}=A_{2} C_{5}+A_{1} C_{6}-\alpha A_{6}+C_{1} A_{5} \text {; } \\
& d_{7}=A_{2} C_{6}+C_{1} A_{6} \quad d_{8}=d_{2} / 42 ; \\
& d_{9}=d_{3} / 30 ; \quad d_{10}=d_{4} / 20 \\
& d_{11}=d_{5} / 12 ; \quad d_{12}=d_{6} / 6 \\
& d_{13}=d_{7} / 2 \quad f_{3}=B_{1} C_{7}+B_{3} f_{1} ; \\
& f_{4}=B_{2} C_{7}+B_{4} f_{1} ; \quad f_{5}=B_{1} C_{8}+B_{3} C_{3} \\
& f_{6}=B_{2} C_{8}+B_{4} C_{3} ; \quad f_{7}=s_{1} C_{7}+s_{4} C_{1} \\
& f_{8}=s_{2} C_{7}+s_{1} C_{8}+B_{5} f_{1}+s_{4} C_{3} ; \\
& f_{9}=s_{3} C_{7}+s_{2} C_{8}+B_{6} f_{1}+B_{5} C_{3}
\end{aligned}
$$




$$
\begin{aligned}
& f_{10}=s_{3} C_{8}+C_{3} B_{6} ; \quad f_{11}=f_{3} / n^{2} ; \\
& l_{23}=\frac{l_{16}}{840} ; \quad l_{24}=\frac{l_{17}}{360} ; \quad l_{25}=\frac{l_{18}}{120} ; \\
& f_{12}=f_{4} / n^{2} \quad f_{13}=-\left(2 \frac{f_{4}}{n^{3}}-\frac{f_{5}}{n^{2}}\right) ; \\
& l_{26}=\frac{l_{19}}{24} ; \quad s_{5}=s_{1} B_{3} n^{2}-B_{1} s_{4} n^{2} ; \\
& f_{14}=-\left(2 \frac{f_{3}}{n^{3}}-\frac{f_{6}}{n^{2}}\right) \quad f_{15}=f_{7} / 20 \\
& s_{6}=s_{1} B_{4} n^{2}-B_{2} s_{4} n^{2} ; \\
& f_{16}=f_{8} / 12 ; \quad f_{17}=f_{9} / 6 ; \\
& s_{7}=\left(s_{2} B_{3} n^{2}-B_{1} B_{5} n^{2}+\beta m^{2} r^{2} h^{3} \frac{\operatorname{Pr}}{k m} f_{12}\right) \\
& f_{18}=f_{10} / 2 \\
& q_{1}=\operatorname{Pr}\left(-d_{8}+d_{9}-d_{10}+d_{11}-d_{12}+d_{3}\right) \\
& q_{2}=\frac{\operatorname{Pr}}{k m}\left(\begin{array}{l}
\left(f_{11}+f_{13}\right) \cosh n+\left(f_{12}+f_{14}\right) \sinh n+ \\
f_{15}+f_{16}+f_{17}+f_{18}
\end{array}\right) \\
& s_{8}=\left(s_{2} B_{4} n^{2}-B_{2} B_{5} n^{2}+\beta m^{2} r^{2} h^{3} \frac{\operatorname{Pr}}{k m} f_{11}\right) \\
& s_{9}=\left(s_{3} B_{3} n^{2}-B_{1} B_{6} n^{2}-B_{1} s_{4} n^{2}-2 s_{1} B_{3}+\right. \\
& \left.\beta m^{2} r^{2} h^{3} \frac{\operatorname{Pr}}{k m}\left(f_{11}+f_{14} n\right)\right)+2 B_{1} s_{4} \\
& q_{3}=\frac{\operatorname{Pr}}{k m}\left(f_{13}\right) ; \quad q_{4}=\frac{k}{h} \frac{\operatorname{Pr}}{k m}\left(f_{14} n+f_{11}\right) \\
& q_{5}=\frac{\left(q_{1}-q_{2}+q_{3}-q_{4}\right) h}{k+h} ; q_{6}=\frac{k q_{5}}{h}+q_{4} ; \\
& q_{7}=-\left(q_{5}+q_{2}\right) ; q_{8}=\left(q_{6}-q_{1}\right) ; l_{13}=G \operatorname{Pr} \frac{d_{2}}{6} \\
& s_{11}=5 f_{15} \beta m^{2} r^{2} h^{3} G \frac{\operatorname{Pr}}{k m} ; s_{12}=4 f_{16} \beta m^{2} r^{2} h^{3} G \frac{\operatorname{Pr}}{k m} \\
& l_{14}=l_{1} A_{3}+12 l_{2} l_{4}-2 l_{1} A_{3}-6 l_{2} l_{4}+G \operatorname{Pr} \frac{d_{3}}{5} \\
& s_{13}=3 f_{17} \beta m^{2} r^{2} h^{3} G \frac{\operatorname{Pr}}{k m} \\
& s_{14}=2 f_{18} \beta m^{2} r^{2} h^{3} G \frac{\mathrm{Pr}}{k m}-2 s_{1} B_{5}+2 s_{2} s_{4} \\
& l_{15}=l_{1} A_{4}+l_{2} A_{3}+12 l_{3} l_{4}-6 l_{1} A_{4}-l_{2} A_{3}-2 l_{3} l_{4}+G \operatorname{Pr} \frac{d_{4}}{4} \\
& s_{15}=-2 s_{1} B_{6}-2 s_{1} s_{4}+\beta m^{2} r^{2} h^{3} G q_{5}+2 s_{3} s_{4} ; s_{16}=\frac{s_{6}}{6 n} ; \\
& l_{16}=l_{2} A_{4}+l_{3} A_{3}+12 A_{1} l_{4}-12 l_{1} A_{5}- \\
& s_{17}=\frac{s_{5}}{6 n} ; \quad s_{18}=-\frac{s_{5}}{4 n^{2}}+\frac{s_{8}}{4 n} ; s_{19}=-\frac{s_{6}}{4 n^{2}}+\frac{s_{7}}{4 n} ; \\
& l_{17}=l_{3} A_{4}+A_{1} A_{3}+12 A_{2} l_{4}-12 l_{1} A_{6}- \\
& 6 l_{2} A_{5}-l_{3} A_{4}+G \operatorname{Pr} \frac{d_{6}}{2} \\
& s_{20}=\frac{s_{6}}{4 n^{3}}-\frac{s_{7}}{4 n^{2}}+\frac{s_{10}}{2 n} \\
& l_{18}=A_{1} A_{4}+A_{2} A_{3}-6 l_{2} A_{6}-2 l_{3} A_{5}+G \operatorname{Pr} d_{7} ; \\
& l_{19}=A_{2} A_{4}-2 l_{3} A_{6}+G q_{6} \\
& s_{21}=\frac{s_{5}}{4 n^{3}}-\frac{s_{8}}{4 n^{2}}+\frac{s_{9}}{2 n} ; \quad s_{22}=-\frac{s_{5}}{8 n^{4}}+\frac{s_{8}}{8 n^{3}} ; \\
& l_{20}=\frac{l_{13}}{5040} ; \quad l_{21}=\frac{l_{14}}{3024} ; \quad l_{22}=\frac{l_{15}}{1680} ; \\
& s_{23}=-\frac{s_{6}}{8 n^{4}}+\frac{s_{7}}{8 n^{3}} ; \quad s_{24}=-\frac{s_{11}}{n^{2}} ; \quad s_{25}=-\frac{s_{12}}{n^{2}} ;
\end{aligned}
$$


$s_{26}=-\frac{12 s_{11}}{n^{4}}-\frac{s_{13}}{n^{2}} ; \quad s_{27}=-\frac{6 s_{12}}{n^{4}}-\frac{s_{14}}{n^{2}}$

$s_{28}=-\frac{24 s_{11}}{n^{6}}-\frac{2 s_{13}}{n^{4}}-\frac{s_{15}}{n^{2}} ; \quad s_{29}=\frac{s_{16}}{n^{2}} ;$

$s_{30}=\frac{s_{17}}{n^{2}} ; \quad s_{31}=-\frac{3 s_{17}}{n^{3}}-\frac{3 s_{17}}{n^{4}}+\frac{s_{18}}{n^{2}} ;$

$s_{32}=-\frac{3 s_{16}}{n^{3}}-\frac{3 s_{16}}{n^{4}}+\frac{s_{19}}{n^{2}}$

$s_{33}=\frac{6 s_{16}}{n^{4}}+\frac{12 s_{16}}{n^{5}}+\frac{6 s_{17}}{n^{4}}-\frac{4 s_{19}}{n^{3}}-\frac{s_{20}}{n^{2}} ;$

$s_{34}=\frac{6 s_{16}}{n^{4}}+\frac{12 s_{17}}{n^{5}}+\frac{6 s_{17}}{n^{4}}-\frac{4 s_{18}}{n^{3}}-\frac{s_{21}}{n^{2}}$

$s_{35}=\frac{-12 s_{16}}{n^{5}}-\frac{6 s_{17}}{n^{5}}-\frac{18 s_{17}}{n^{6}}+\frac{6 s_{18}}{n^{4}}-\frac{2 s_{21}}{n^{3}}+\frac{s_{22}}{n^{2}}$

$s_{36}=\frac{-12 s_{17}}{n^{5}}-\frac{6 s_{16}}{n^{5}}-\frac{18 s_{16}}{n^{6}}+\frac{6 s_{19}}{n^{4}}-\frac{2 s_{20}}{n^{3}}+\frac{s_{23}}{n^{2}}$

$s_{37}=\frac{s_{24}}{30} ; s_{38}=\frac{s_{25}}{20} ; \quad s_{39}=\frac{s_{26}}{12} ; s_{40}=\frac{s_{27}}{6}$

$s_{41}=\frac{s_{28}}{2}$

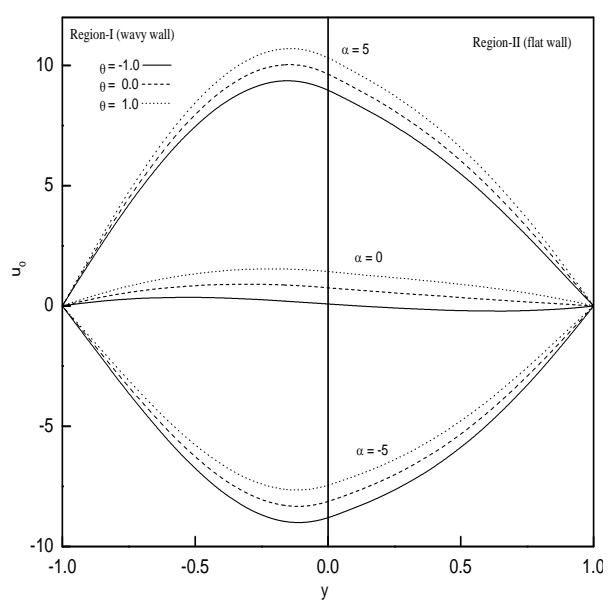

Fig. 3 Zeroth order velocity profiles for different values of $a$

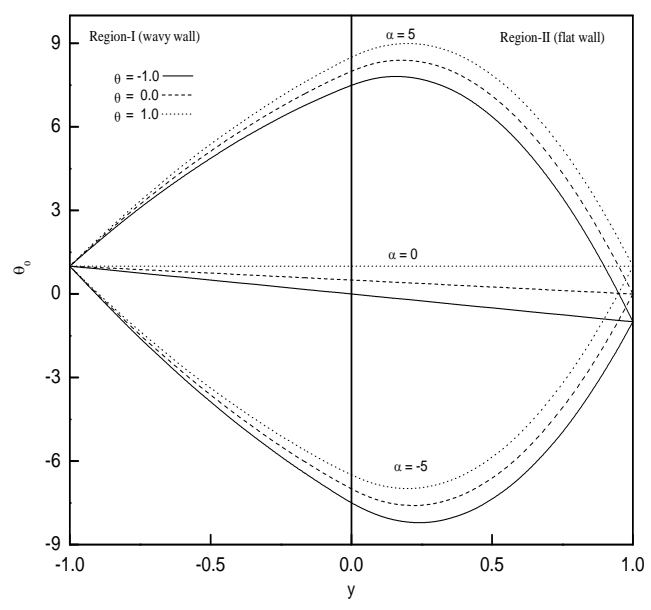

Fig. 4 Zeroth order temperature profiles for different values of $\alpha$

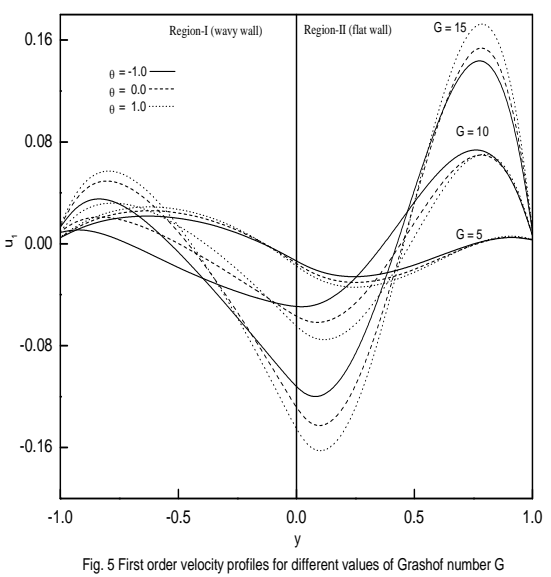



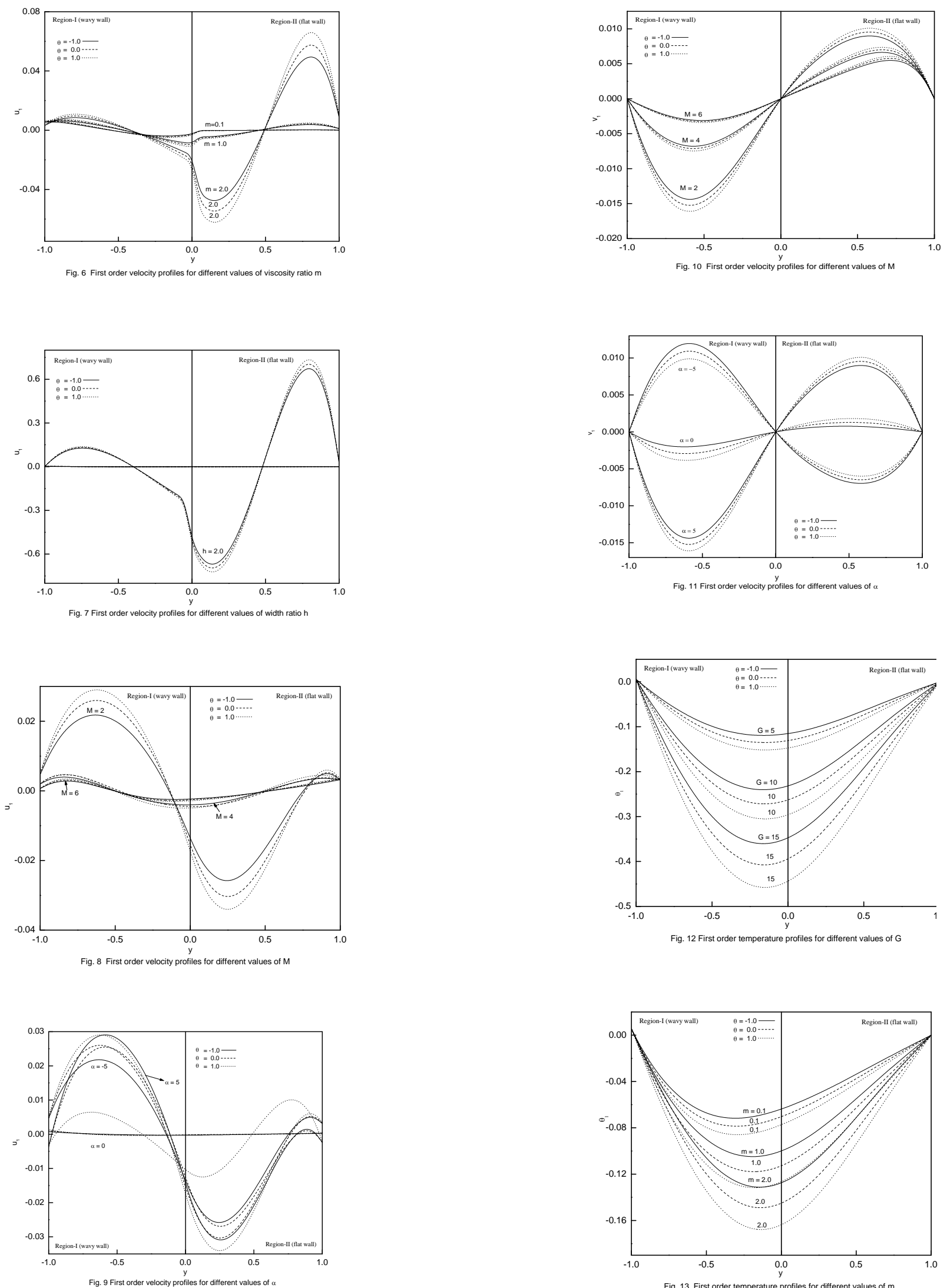

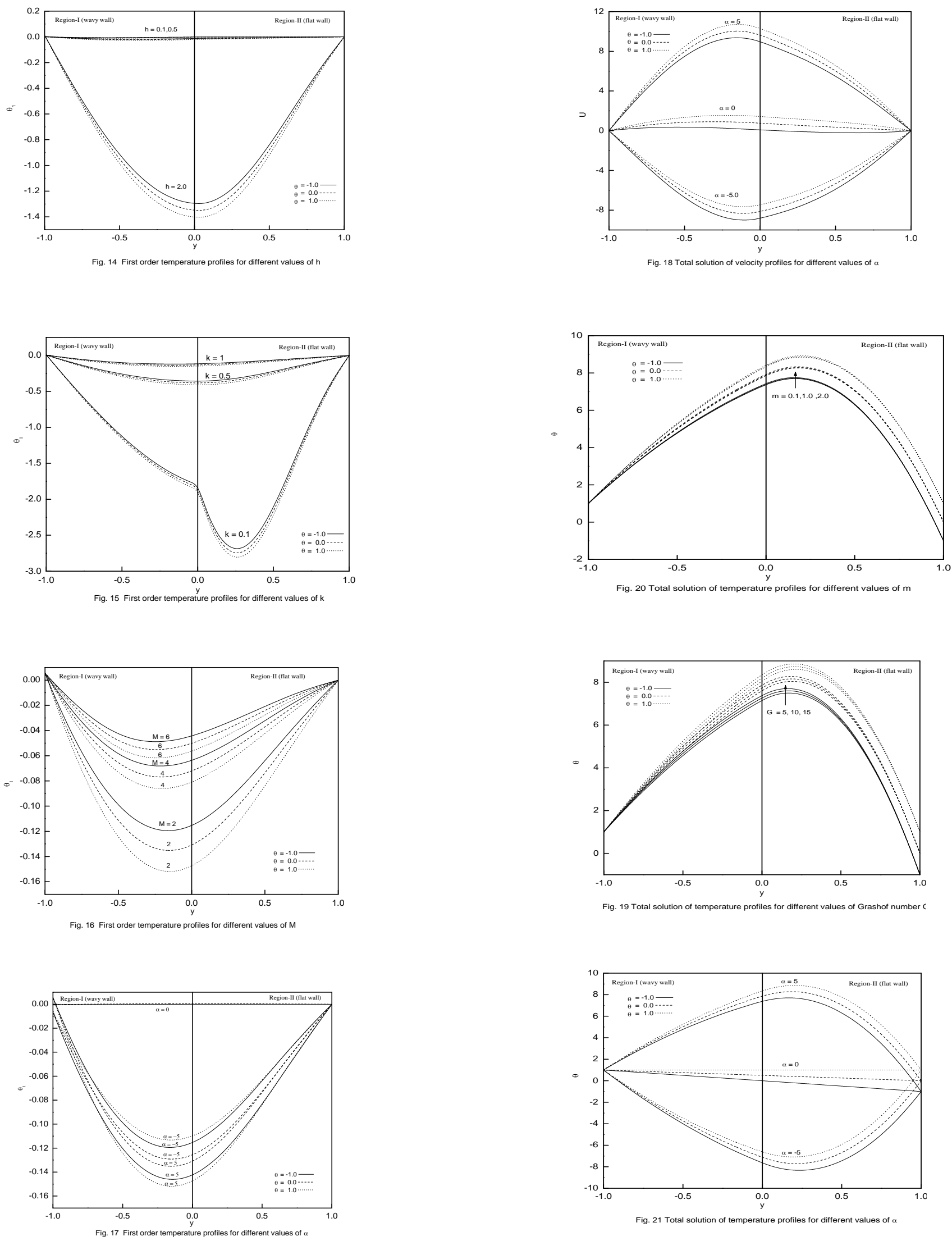


\section{REFERENCES:}

[1]. Sathe S., Sammkia B., (1998) A review of recent developments in same practical aspects of air-cooled electronic packages, Trans. ASME. J. Heat Transfer vol. 120 pp 830-839.

[2]. London M.D., Campo A., (1999) Optimal shape for laminar natural convective cavities containing air and heated from the side, Int. Comm. Heat mass Transfer vol. 26, pp 389-398.

[3]. Da silva A.K, Lorente S, Bejan A,(2004) Optimal distribution of discrete heat sources on a wall with natural convection, Int. J. Heat Mass Transfer vol. 47 pp 203-214.

[4]. Burns J.C, Parks T, J. (1967) Fluid Mech.29 405-416

[5]. Wang G. Vanka P. (1995) Convective heat transfer in periodic wavy passages. Int. J. Heat Mass Transfer vol. 38(17) pp 3219

[6]. Yao L.S., (1983) Natural convection along a vertical wavy surface, ASME J. Heat Transfer vol. 105 pp 465468.

[7]. Moulic S. G., Yao L.S., (1989) Mixed convection along a wavy surface, ASME J. Heat transfer vol.111 pp 974-979.

[8]. Lohrasbi. J and Sahai.V (1988) Magnetohydrodynamic Heat Transfer in two-phase flow between parallel plates. Applied scientific Research vol. 45 pp. 53-66.

[9]. Malashetty M.S. and Umavathi J.C., (1997) Twophase Magnetohydrodynamic flow and heat transfer in an inclined channel, Int. J. Multiphase Flow, vol. 22, pp 545-560.

[10]. Malashetty M. S, Umavathi J. C. and Prathap Kumar (2000) Two-fluid magneto convection flow in $n$ inclined channel" I.J. Trans phenomena, vol. 3. Pp 7384.

[11]. Malashetty M. S, Umavathi J. C, Prathap Kumar J. (2001) Convection magnetohydrodynamic two fluid flow and heat transfer, vol.37, pp 259-264.

[12]. Chamkha Ali. J, (2000) Flow of two- immiscible fluids in porous and nonporous channels, Journal of Fluids Engineering, vol. 122, pp 117-124. 